\title{
Panorama de la cryothérapie
}

La cryothérapie est une technique très utilisée dans le domaine du sport. Les effets bénéfiques du froid sont nombreux : analgésique, vasomoteur, anti-inflammatoire, myorelexant. Les techniques sont diverses, allant de la simple vessie de glace aux chambres de cryothérapie corps entier. Cette dernière nécessite un certificat de noncontre-indication et un accompagnement par du personnel médical qualifié.

Mots clés - analgésie ; bombe de froid ; cryothérapie corps entier

Overview of cryotherapy. Cryotherapy is a technique frequently used in the field of sport. The beneficial effects of cold are numerous: analgesic, vasomotor, anti-inflammatory, muscle relaxant. There are various techniques, ranging from the simple ice bag to whole body cryotherapy chambers. The latter require a certificate of non-contraindication and support from qualified medical staff.

Keywords - analgesia; cold spray; whole body cryotherapy

acryothérapie est le traitement par le froid, quelle que soit la technique appliquée sur le corps, qui provoque une réduction de la température locale du corps (tableau 1) [1]. Elle est surtout utilisée pour calmer les douleurs liées à des pathologies d'entorses, de tendinites, de lésions musculaires. Très fréquente sur les terrains de sport, la bombe de froid est largement utilisée pourcalmerladouleursuiteàunchoc.

\section{Historique}

L'application thérapeutique du froid est très ancienne, puisque Hippocrate l'utilisait 460 ans avant J.-C. pour soulager les douleurs et réduire les œdèmes.

Redécouvert dans les années 1950, abandonné puis repris dans les années 1970, le concept est désormais largement utilisé dans le milieu sportif mais aussi dans d'autres applications comme la protection du cerveau lors de situations d'urgence cardiaque ou cérébrale. Afin d'évaluer de façon codifiée des résultats obtenus par l'abaissement de la température cutanée, les Japonais, puis les Arabes, ont élaboré uneméthodologiescientifique.

\section{Physiologie}

L'application du froid entraîne de nombreux effets positifs : analgésique, vasoconstricteur local,
Alain Frey

Chef du département

médical

INSEP, département médical 11, avenue du Tremblay, 75012 Paris, France

\section{Tableau1.Principalestempératuresobtenuesaprèsapplication defroid.}

\begin{tabular}{|c|c|c|c|}
\hline & Pochedeglace(10minutes) & $\begin{array}{l}\text { Immersioneneaufroide } \\
\left(8-10^{\circ} \mathrm{C} ; 4-5 \text { minutes }\right)\end{array}$ & $\begin{array}{l}\text { Cryothérapie corps entier } \\
\text { (3minutes) }\end{array}$ \\
\hline Température de la peau & $\begin{array}{l}-18^{\mathrm{a}} \\
-20 \\
-20^{\mathrm{a}} \\
-20 \\
-22^{\mathrm{a}} \\
-25,7-26,4\end{array}$ & $\begin{array}{l}-6,2(0,5) \\
-8,4(0,7) \\
-9,0(0,8)\end{array}$ & $\begin{array}{l}-2,5-8,7 \\
-6,7^{\mathrm{a}} \\
-8,1(0,4) \\
-12,1(1,0) \\
-10,3(0,6) \\
-13,7(0,7) \\
-19,4^{\mathrm{a}}\end{array}$ \\
\hline $\begin{array}{l}\text { Température intramusculaire }(2 \mathrm{~cm} \text { de } \\
\text { profondeur) }\end{array}$ & $\begin{array}{l}-1,76(1,37) \\
-2,0 \\
-2,0^{\mathrm{a}} \\
-2,7^{\mathrm{a}} \\
-3,88(1,83)\end{array}$ & $-1,7(0,9)$ & $-1,2(0,7)$ \\
\hline Température centrale & $\begin{array}{l}0 \\
0\end{array}$ & $\begin{array}{l}-0,2(0,1) \\
-0,4(0,2)\end{array}$ & $\begin{array}{l}0 \\
-0,3(0,2)\end{array}$ \\
\hline
\end{tabular}

Toutesles valeurs sonten ${ }^{\circ} \mathrm{C}$.

${ }^{a}$ Déviation standard non renseignée.

Source : d'après Bleakley CM, Bieuzen F,Davison GW, Costello JT.Whole-body cryotherapy: empirical evidence and theoretical perspectives. Open Access Journal of Sports Medicine.2014:5:25-36.
Adresse e-mail : alain.frey@insep.fr (A. Frey). 
réduction des œdèmes, perméabilité vasculaire, diminution du métabolisme musculaire et diminution de la réponse inflammatoire suite à des dommages musculaires. C'est probablement cet ensemble de facteurs qui permet de participer à la récupération des performances physiques.

\section{Effet analgésique}

Sous l'effet du froid, la vitesse de conduction de l'information au cerveau est abaissée et entraîne une réduction immédiate de la douleur. Cetteapplication de froid permet de diminuer le ressenti de la douleur périphérique pour des durées minimales de trois heures voire dans certainscas de lafairedisparaîtreen cas de traumatismes très bénins.

Sila douleur mécanique persisteou récidive, il convient de s'assurer de l'origine exacte de la douleur et de rechercher une pathologie inflammatoire, infectieuse, vasculaire ou neurologique[2].

\section{Effet vasomoteur}

Les phénomènes vasomoteurs vont entraîner une réponse circulatoire souvent importante, surtout si la vasodilatation touche en profondeur les tissus. Deux actions sont possibles : l'une va jouer sur la circulation sanguine et l'autre sur la circulation lymphatique. La première réponse est la constriction des artérioles et veinules (moins de 15 minutes après). Le débit sanguin dans cette zone diminue afin que le corps puisse conserver une températurecentralenormale.

La vasodilatation peut être induite par le froid après une période initiale de vasoconstriction si le froid se maintient pendant plus d'environ 15 minutes ou si la température cutanée descend sous les $10^{\circ} \mathrm{C}$.

Cette réaction appelée la "Hunting reaction" [3] est prédominante dans les zones distales où les anastomoses artérioveineuses sont très fréquentes sous la peau. II a été prouvé que cette réponse ne se produit pas dans les tissus profonds. Après l'arrêt de l'exposition au froid, la température des parties du corps adjacentesaugmente.

Par exemple, en cas d'utilisation de la cryothérapie gazeuse, les effets vasomoteurs observés peuvent varier en fonction de l'action en profondeur sur les tissus exposés à ce type de froid. Au niveau superficiel, on décrit essentiellement trois actions :

- une vasoconstriction réflexe immédiate;

- une vasodilatation cutanée pouvant atteindre plus de $100 \%$ en 20 secondes (alors que l'application de froid traditionnel entraîne $80 \%$ après 20 minutes) ;

- une normalisation des perturbations de la microcirculation veinoartériolaire présentes lors des syndromes douloureux régionaux complexes.

Sous l'effet de la pression du gaz utilisé, ces trois actions combinées provoquent une augmentation de la pression partielle en oxygène des tissus et diminuent la pression partielle en $\mathrm{CO}_{2}$. Ces phénomènes produiraient un massage et un drainage des œdèmes, baisseraient la stase au sein des capillaires et augmenteraient le débit circulatoire afin de diminuer la quantité d'histamine, d'acide lactique et de fibrine produit localement en cas d'agression de l'organisme.

\section{Effet anti-inflammatoire}

Sous l'effet du choc thermique provoqué par l'application du froid, on retrouve une inhibition de l'action des enzymes responsables de l'inflammation, ce qui peut diminuer voire arrêter instantanément et durablement la crise inflammatoire. Ces actions sont expliquées principalement par la mise en place d'une régulation de la circulation sanguine limitant ainsi la production enzymatique et par la baisse de la synthèse de certaines métalloprotéases.
En cas de poussée inflammatoire, les capacités de lutte de l'organisme sont dépassées et peuvent ainsi favoriser la majoration des signes cliniques locaux sous l'effet d'une production accrue des métalloprotéases (collagénases, élastases, hyaluronidases) pouvant provoquer jusqu'à la nécrose des tissus touchés.

Ces métalloprotéases sont très thermosensibles et ne se synthétisent plus sous l'action du froid.

\section{Phase vasculaire}

Face à une agression quel que soit son type, l'organisme déclenche sa propre réaction inflammatoire naturelle. II ne faut pas empêcher cette défense naturelle de l'organisme mais plutôt l'accompagner ce qui veut dire sur le plan thérapeutique de ne pas prescrire d'anti-inflammatoires à la phase initiale d'un traumatisme.

Les enzymes de l'inflammation, libérés lors de cette réaction naturelle de l'organisme, entraînent une dilatation vasculaire facilitant l'arrivée des lymphocytes puis des macrophages permettant à l'organisme de lutter contre l'inflammation quelle qu'en soit l'origine. II apparaît alors classiquement la triade des signes cliniques ressentis par le patient à savoir rougeur, chaleur et douleur.

\section{Effet myorelaxant}

II est constaté que la fibre musculaire se relâcherait sous l'effet de l'application locale d'une très basse température. Le mécanisme physiologique complexe de cet effet n'est pas entièrement expliqué. Des études en cours par élastographie musculaire pourraient nous apporter des informations utiles sur les effets réels de l'application de froid en cas de traumatismes et pourraient modifier les protocoles actuels d'utilisation de la cryothérapie sur le terrain et lors des soins en cabinet. 
Sur le plan neurologique, la stimulation par le froid des voies réflexes médullaires pourraient inhiber l'action des motoneurones gamma et faciliter ainsi une réduction du tonus musculaire.

\section{Autres effets}

D'autres effets peuvent être enregistrés:

- une action hémostatique qui diminuele saignement;

- le refroidissement influe sur certains paramètres endocriniens et indicateurs de dommages cellulaires. De ce fait, les médiateurs de l'inflammation (prostaglandines, leukotriènes...) sont d'une grande efficacité ;

- spasticité réduite des tissus. Une réduction de l'activité des motoneurones provoquée par l'excitation des afférences cutanées entraîne la réduction de la spasticité. Elle intervient en réduisant la décharge afférente de l'axe.

\section{Mécanismes d'action de certainestechniques Laglace}

L'application locale de froid en utilisant la glace nécessite quelques précautions d'usage afin d'éviter de brûler la peau. Cette action se fait principalement par conduction du froidentrelapochedeglaceetlapeau mais eninterposantun linge. La poche de glace contient de l'eau froide associée à des glaçons afin d'obtenir un froiduniformesurlapeau.

\section{L'immersion en eau froide}

S'ajoutent au froid les effets physiologiques de la pression hydrostatique et de la relaxation. Cette pression facilite la réduction des œdèmes locaux participant à la diminution des phénomènesdouloureux.

La relaxation induite par l'utilisation de cette technique peut favoriser la diminution de la sensation de fatigue générée par l'effort intensif pratiqué.

\section{L'aérothérapie}

Cette technique utilise des compresseurs à air qui fonctionnent avec une pression de sortie inférieure à 1 bar ne générant pas de microcristallisation en sortie de buse. Ce refroidissement par soufflage d'air froid agit par convection.

\section{La neurocryostimulation}

C'est une technique sonique dont la pression d'éjection du jet est largement supérieure à 1 bar [4]. Le gaz est expulsé sous forme de microcristaux de neige carbonique à $78{ }^{\circ} \mathrm{C}$ avec une pression aux alentours de 2,2 bars sur la peau en respectant les consignes d'utilisation. Ce choc thermique s'effectue parconvectiongrâce au phénomène de sublimation (passage direct de l'étatsolideà l'état gazeux).

Dans l'utilisation de cette technique, nous retrouvons l'association de trois actions simultanées favorisant ce choc thermique local :

- un froid à très basse température $\left(-78^{\circ} \mathrm{C}\right)$;

- une vitesse de refroidissement liée à la pression du gaz (50 bars à $15^{\circ} \mathrm{C}$ );

- une onde de choc de $400 \mathrm{~Hz}$ à l'expulsion des microcristaux dans le cône d'éjection du jet.

Lors de l'utilisation de la cryothérapie corps entier (CCE) (tableau 2), nous retrouvons un certain nombre d'effets systémiques bien décrits dans la littératurecommelechocthermique[4,5]:

- une augmentation du débit cardiaque et une meilleure vascularisation des tissus;

- une réponse endocrinienne hormonale (surrénales, thyroïde, axe corticotrope...);

- un effet anti-inflammatoire et des modifications immunitaires ;

- un contrôle de la thermorégulation par récepteurs cutanés et des organes internes;

- une régulation au niveau de l'hypothalamus par l'activation de neurotransmetteurs;

- une réponse neurovégétative du cortex à distance avec action sur les systèmes noradrénergiques et sérotoninergiques;

- une libération de noradrénaline entraînant une augmentation de la fréquence cardiaque et de la pression artérielle, une vasoconstriction périphérique vasculaire diminuant la perte de chaleur, une bronchodilatation par stimulation dusystèmenerveuxsympathique;

- une modification des neurotransmetteurs avec diminution de la vitesse de transmission des influx nerveux participant à la réduction de la douleur et une diminution des messages nociceptifs à cause de la diminution des médiateurs de l'inflammation;

- une modification du statut oxydant avec, au repos, une augmentation du statut antioxydant et, à l'effort, une diminution du stress oxydatif ; cela favorise une meilleure récupérationmusculaire.

Tableau2. Résumédeseffets de la cryothérapie corps entier [1].

\begin{tabular}{lllll} 
Température des tissus & & & Inflammation & \multicolumn{2}{c}{ Dommages musculaires } \\
\cline { 1 - 3 } Peau & Muscle & Centrale & & \\
\hline+++ & + & $\varnothing$ & ++ & $\varnothing$ \\
\hline Récupération fonctionnelle & Récupération perçue & Réhabilitation & Stress oxydatif & Système nerveuxautonome \\
\hline$+/ \varnothing$ & + & + & ++ & ++ \\
\hline
\end{tabular}




\section{Références}

[1] BleakleyCM, Bieuzen F, Davison GW, Costello JT.

Whole-body cryotherapy: empirical evidence and theoretical perspectives. Open

Access Journal of Sports Medicine.2014;5:25-36

[2] Hubbard TJ, Denegar CR. Does cryotherapy improve outcomes with soft tissue injury? J Athl Train. 2004;39(3):278-9.

[3] Daanen HAM, Van de Linde FJG, Romet TT. The effect of body temperature on the hunting response of the middle finger skin temperature. Eur J Appl Physiol. 1997;76:538-43.

[4] Mesure S, CatherinMarcel B, Bertrand D. La cryothérapie corps entier : littérature et perspectives de recherches. KinesitherRev. 2014;14(152-3):56-60.

[5] Pournot H, Bieuzen F, Louis J, Mounier R, Fillard JR, Barbiche E, et al. Time course of changes in inflammatory after whole-body cryotherapy multi exposures following severeexercise.Plos One. 2011;6(7):e22748.

[6] Hausswirth C. Sport et chaleur: l'apport des vestes réfrigérantes Médecins du Sport. 2009;94:25-6.

[7] Webb NP, Harris NK, Cronin JB, Walka C. The relative efficacy of three recoverymodalities after professionalrugbyleague matches. J Strength Cond Res. 2013;27(9):2449-55.

[8] Gregson W, Black MA, Jones H, Milson J, Morton J, Dawson B, et al. Influence of cold water immersion on limb and cutaneous blood flow at rest. Am J Sports Med. 2011;39(6):1316-23.

[9] Wegmann M,

Faude O, PoppendieckW Hecksteden A, Fröhlich M,

Meyer T, et al. Pre-cooling and sports performance: a metaanalytical review. Sports Med. 2012;42(7):545-64

[10] Adam J. Impact de la cryothérapie corps entier sur larécupération musculaire chezle sportif. Kinesither Rev. 2014;14(152-153):61-5.

[11] Schaal K, Le Meur Y, Louis J, Filliard JR, Hellard P,Casazza G, et al. WholeBodyCryostimulation
Les différentsmoyens d'application thérapeutique dufroid Vessie de glace

La vessie est remplie avec de l'eau froide dans laquelle on place des glaçonsoudelaglacepiléeafind'obtenir l'application d'un froid homogène. II faut placer un linge humide pour conduire le froid en profondeur et augmenter ainsi la durée d'efficacité tout en permettant de protéger la peau (figure 1).

\section{Bombedefroid}

La bombe (figure 2) renferme un gaz et permet de pulvériser du froid sur une contusion directe ou une lésion de l'appareil locomoteur en dehors de toute plaie cutanée.

II esrt important de respecter une certaine distance par rapport à la peau et surtout de ne pas rester trop longtemps sur la zone afin d'éviter les brûlures cutanées.

\section{Poche instantanée à usage unique}

Les poches instantanées à usage unique (figure 3) sont pratiques quand on ne dispose pas de glace immédiatement. Soit il s'agit de poche contenant un sel et de l'eau séparés. En rompant cette séparation, la réaction chimique refroidit spontanément la poche. On l'applique sur la zone blessée, avec une protection intermédiaire.

Soit il s'agit de poche contenant un gel et, dans ce cas, la température du gel au départ du congélateur est entre $-11^{\circ} \mathrm{C}$ et $-18^{\circ} \mathrm{C}$, mais l'effet thermique est moindre que la glace car le gel épouse moins bien la surface de la zone lésée.

Dans tous les cas, il faut protéger la peau avec un linge afin d'éviter les brûlures.

\section{Les cryovestes}

Les cryovestes (figure 4) ont été validées par l'Institut de médecine navale à Toulon (83) avant les Jeux olympiques de Pékin d'été en 2008

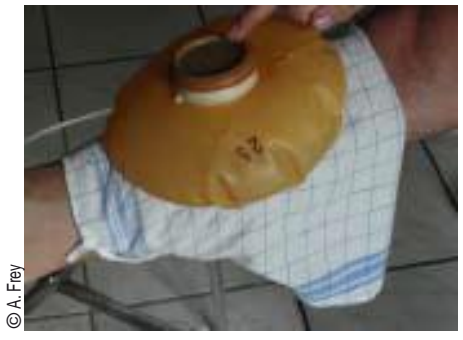

Figure 1. Vessie de glace.

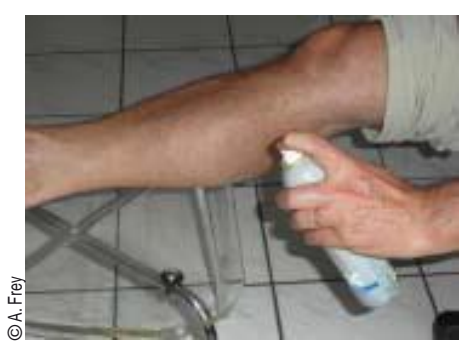

Figure 2. Bombe de froid.

[6]. Elles ont été mises au point à partir de données enregistrées dans une chambre climatique où les températures et hygrométries de Pékin étaient simulées $\left(30^{\circ} \mathrm{C}\right.$ et $80 \%$ d'humidité)

Les cryovestes permettent aux sportifs de se refroidir de manière diffuse en jouant principalement sur le tronc ; elles conservent le froid grâce à un système de protection en téflon intégré dans la veste et dans lequel se logent les poches de neige en regard de la face antérieure et postérieure du tronc. Ces poches de neige sont placées dans un congélateur avant utilisation.

L'avantage de ces vestes consiste en un refroidissement modéré du corps aux alentours de $20^{\circ} \mathrm{C}$ sans altérer ni les échanges thermiques type sudation et évaporation de la chaleur permettant à la peau de "respirer", ni la fréquence cardiaque du sportif.

\section{Bains d'eauglacée}

Les bains d'eau glacée sont couramment utilisés dans les sports d'équipe, avec comme principe général l'immersion soit d'une partie du corps (les membres inférieurs),

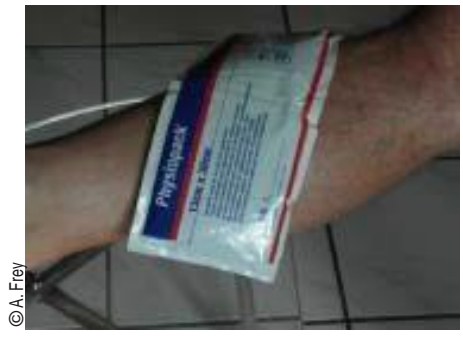

Figure3.Gel (pocheinstantanée à usage unique).

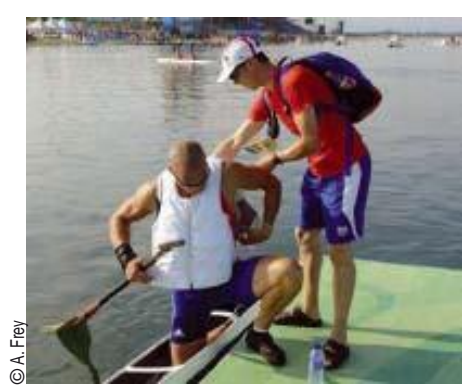

Figure 4. Cryoveste.

soit la totalité du corps. Cela peut se réaliser dans une simple poubelle ou alors dans des bains dédiés d'eau très froide. Ces techniques montrent des résultats intéressants dans la littérature scientifique comme l'hydrothérapie contrastée [7,8].

\section{F L'immersiondansl'eaufroide} suit un protocole : immersion du corps d'un sportif (ou partie) dans un bain d'eau froide entre 9 et $12^{\circ} \mathrm{C}$ pendant environ 14 à 15 minutes.

F L'hydrothérapiecontrastéese déroule de la manière suivante : un premier bain d'eau chaude aux alentours de $38-39{ }^{\circ} \mathrm{C}$ suivi d'un bain froid à $9-12{ }^{\circ} \mathrm{C}$ d'une durée de 15 minutes.

Plusieurs études dans les sports d'équipe (football, rugby) montrent un avantage à la première technique avec une plus grande capacité à réduire les œdèmes et les dommages causés aux muscles.

\section{Cryothérapie gazeuse ou àairfroid}

F Laneurocryostimulation(ou cryothérapie gazeuse hyperbare), propulse un froid sec dont la tolérance est bien acceptée par le 
patient. Son application est de 20 minutes, avec une possible répétition deux heures après, mais il n'existe aucune preuve scientifique. Lors de l'utilisation de cette technique, la température cutanée chute en moins de 30 secondes et, en 2 minutes de traitement, on obtient le même type de refroidissement que l'application d'une vessie de glace pendant 20 minutes.

$\mathrm{F}$ La cryothérapie à air froid est un modèle plus économique par absence de consommable et entraîne moins de risques de lésions cutanées. Cette technique utilise l'air ambiant aspiré et refroidi en passant dans un réservoir de froid permettant d'atteindre environ $-300^{\circ} \mathrm{C}$. Cet air froid est ensuite propulsé sur la peau du sportif mais à une pression moins forte que dans la technique précédente, ce qui nécessite une application plus longue pour espérer obtenir le même effet.

\section{Cryothérapie corps entier ou corps partiel}

Sont apparues récemment les chambres de cryothérapie corps entier(CCE)(figure5)permettantde descendre à des températures oscillantentre $-110^{\circ} \mathrm{Cet}-140^{\circ} \mathrm{C}$. LesCCEutilisentdescompresseurs refroidissant l'air alors que les cryothérapies corps partiel (CCP), où la tête est en dehors, utilisentl'azote.

Ces chambres sont de plus en plus utilisées par les sportifs mais nécessitent une surveillance par un personnelqualifiémédical ou paramédical et coûtentrelativementcher.

Elles permettent de produire les effets mais aussi de jouer sur la récupération en améliorant la qualité du sommeil. En revanche, le froid produit localement est moins marqué que l'application locale de glace.

\section{Contre-indications}

Les contre-indications sont multiples et diffèrent selon les techniques utilisées.



Figure5. Chambre de cryothérapie corps entier de l'INSEP.

Pour les techniques utilisant le corps entier, le médecin effectue une visite de non-contre-indication et délivre un certificat autorisant le sportif à utiliser la CCE par exemple. Les contre-indications suivantes sont recherchées:

- une allergie a u froid ;

- un syndrome de Raynaud : le refroidissement des extrémités(mainset pieds) peut être très gênanten cas detempératurebasse;

- des lésions cutanées comme des plaies multiples ou une chirurgie récente;

- une cryoglobulinémie;

- une hypertension artérielle non contrôlée, affection cardiovasculaireévolutive;

- une pathologie pulmonaire sévère;

- le port de piercing ou de lentilles;

- le port d'un pacemaker;

- unegrossesse;

- être mineur : il existe actuellement un principe de précaution édicté par la Société française de pédiatrie (SFP) et la Société française de médecine de l'exercice et du sport (SFMES).

Pour les techniques locales, il existe peu de contre-indications en dehors de plaies, à condition de respecter les conditions d'utilisation de chaque technique pour surtout éviter les brûlures cutanées.

\section{Indications actuelles}

Les indications sont nombreuses au vu des effets physiologiques présentés:

- en traumatologie du sport (contusion, entorse, lésion musculaire...);
- en entraînement pour favoriser la récupération et la performance [9-11];

- en rhumatologie [12] : rhumatologie inflammatoire chronique (polyarthrite rhumatoïde, spondylarthrite ankylosante, fibromyalgie...), rhumatologie abarticulaire (tendinite, bursite, ténosynovite, périostite...), rhumatologie dégénérative (traitement de la douleur).

\section{Conclusion}

L'application de froid ou cryothérapie est un moyen efficace à utiliser dans le cadre des prises en charge des blessures chez le sportif en y associant d'autres techniques comme la compression, la décharge du membre inférieur et le drainage par exemple[13].

Les sportifs de haut niveau, disposant au sein de leur structure d'une cryothérapie corps entier, l'utilisent régulièrement après des séances intensives d'entraînement ou de compétitions rapprochées pour favoriser la récupération musculaire en limitant les processus inflammatoires liés à la microdestruction des cellulesmusculaires.

En dehors des moyens locaux de cryothérapie qui feront toujours partie de l'arsenal thérapeutique même si les protocoles restent à affiner car ils s'avèrent souvent empiriques [14] -, l'utilisation des technologies plus sophistiquées comme la CCE se développe, mais nécessite de poursuivre I a recherche afin de clarifier les effets réels comme dans le cas de la CCE. Pour cela, a été créée la Société française de cryothérapie corps entier dont le but principal est de promouvoir la recherche et de mettre en place des protocoles d'utilisation de cette technique aussi bien dans le domaine du sport que dans les spécialités où existent des maladies inflammatoires chroniques, surtout en rhumatologie. w

\section{Références}

Limits Overreaching in Elite Synchronized Swimmers. Med Sci Sports Exerc. 2015 Jul;47(7):1416-25.

[12] Guillot X, Tordi N, Mourot L, Demougeot C, Dugué $\mathrm{B}$, Praté $\mathrm{C}$, et al. Cryotherapyininflammatory rheumaticdiseases: a systematic review. ExpertRev Clin Immunol 2014;10(2):281-94.

[13] Van den Bekerom MP, Struijs PA, Blankevoort L, Welling $L$, van Dijck CN, Kerkhoffs GM, et al. What is the evidence for rest, ice, compression, and elevation therapy in the treatment of ankle sprains in adults? J Athl Train. 2012;47(4):435-43.

[14] Bleakley CM, McDonoughSM, MacAuley DC, Bjordal J. Cryotherapyfor acute ankle sprains:a randomised controlled study of two different icing protocols. $\mathrm{Br} \mathrm{J}$ SportsMed.2006;40(8):700-5.

Déclarationdeliensd'intérêts L'auteurn'a pas transmis de déclarationdeliens d'intérêts en relationaveccetarticle. 